\title{
Becchi-Rouet-Stora-Tyutin-Lagrangian Double Copy of Yang-Mills Theory
}

\author{
Leron Borsten $\odot,{ }^{1, *}$ Branislav Jurčo $\odot,{ }^{2, \dagger}$ Hyungrok Kim, ${ }^{1, \$}$ Tommaso Macrelli $\oplus^{3, \S}$ \\ Christian Saemann $\odot,{ }^{1, \|}$ and Martin Wolf ${ }^{3, \pi}$ \\ ${ }^{1}$ Maxwell Institute for Mathematical Sciences and Department of Mathematics, Heriot-Watt University, \\ Edinburgh EH14 4AS, United Kingdom \\ ${ }^{2}$ Charles University Prague Faculty of Mathematics and Physics, Mathematical Institute, Prague 186 75, Czech Republic \\ ${ }^{3}$ Department of Mathematics, University of Surrey, Guildford GU2 7XH, United Kingdom
}

(Received 4 November 2020; accepted 8 April 2021; published 12 May 2021)

\begin{abstract}
We show that the double copy of gauge theory amplitudes to $\mathcal{N}=0$ supergravity amplitudes extends from tree level to loop level. We first explain that color-kinematics duality is a condition for the BecchiRouet-Stora-Tyutin operator and the action of a field theory with cubic interaction terms to double copy to a consistent gauge theory. We then apply this argument to Yang-Mills theory, where color-kinematics duality is known to be satisfied on shell at the tree level. Finally, we show that the latter restriction can only lead to terms that can be absorbed in a sequence of field redefinitions, rendering the double copied action equivalent to $\mathcal{N}=0$ supergravity.
\end{abstract}

DOI: 10.1103/PhysRevLett.126.191601

Introduction and summary.-Yang-Mills scattering amplitudes have been conjectured to satisfy a colorkinematics (CK) duality [1-3]: each amplitude can be written as a sum over purely trivalent graphs such that the kinematical numerators satisfy the same antisymmetry and Jacobi identities as the color contributions. CK duality has been shown to hold at the tree level [4-12]. If it holds, replacing the color contributions of a Yang-Mills amplitude with another copy of the kinematical contributions yields a gravity amplitude [3]. This is known as the double copy prescription, and it has far reaching consequences for our understanding of quantum gravity. For reviews and references see Refs. [13-15].

Explicit nontrivial examples [2,16-36] have suggested that the double copy extends to the loop level (i.e., to the integrands of loop amplitudes). In this Letter, we argue that this is indeed the case to any finite loop order.

Our approach builds on the ideas of manifestly CK-dual classical kinematic structure constants and Lagrangians [3,37-44]. A key ingredient in our argument is the BecchiRouet-Stora-Tyutin (BRST) formalism and its enlarged field space of external states [45]. We extend the idea that the BRST framework can be double copied [44,46-51] and double copy the complete BRST Lagrangian. See also Ref. [32] for a powerful approach to loop-level CK-dual

Published by the American Physical Society under the terms of the Creative Commons Attribution 4.0 International license. Further distribution of this work must maintain attribution to the author(s) and the published article's title, journal citation, and DOI. Funded by SCOAP . amplitudes using the BRST invariance of the underlying pure spinor superstring.

We make the crucial observation that on-shell, CK duality violations due to longitudinal gluon modes can be compensated by harmless field redefinitions of the NakanishiLautrup (NL) field. The Ward identities of the BRST symmetry then allow us to transfer CK duality from gluon amplitudes to those involving ghosts. Finally, on-shell treelevel CK duality on the BRST-extended field space turns out to suffice to show that the BRST-Lagrangian double copied theory provides the loop integrands of a consistent perturbative quantization of $\mathcal{N}=0$ supergravity. We stress that our results do not imply or rely on loop-level CK duality.

A longer paper giving explicit expressions for many of the steps discussed only abstractly in the following and explaining the origin of the double copy in terms of homotopy algebras, mathematical objects unifying scattering amplitudes and BRST Lagrangians, is in preparation [52]. There, we also intend to make a connection to the observed nontrivial modifications of CK duality at the loop level, cf., e.g., Refs. [53,54].

The BRST-Lagrangian double copy.-We start with an abstract perspective on the double copy. Any Lagrangian field theory is equivalent to a field theory with exclusively cubic interaction terms, by blowing up higher order vertices using auxiliary fields, cf. also Refs. [55,56]. A generic cubic action is

$$
S=\frac{1}{2} \Phi^{I} \mathfrak{g}_{I J} \Phi^{J}+\frac{1}{3 !} \Phi^{I} \mathfrak{\mathfrak { f }}_{I J K} \Phi^{J} \Phi^{K}
$$

where the fields $\Phi^{I}$ are elements of some field space $\mathfrak{F}$ and the DeWitt index $I$ encodes all field labels (including 
position $x$ ). Summation and space-time integration over repeated indices are understood. We are interested in theories invariant under a gauge symmetry described by a BRST operator $Q$.

It is not hard to see that by blowing up ghost vertices in the Batalin-Vilkovisky (BV) action before gauge fixing, one can always reduce the gauge transformations of all fields to be at most cubic in the fields:

$$
Q \Phi^{I}=\mathfrak{q}_{J}^{I} \Phi^{J}+\frac{1}{2} \mathfrak{q}_{J K}^{I} \Phi^{J} \Phi^{K}+\frac{1}{3 !} \mathfrak{q}_{J K L}^{I} \Phi^{J} \Phi^{K} \Phi^{L} .
$$

We further require that fields split into "left" and "right" components (with independent left and right ghost numbers), but over a common space-time point. Consequently, we expand the DeWitt indices as $I=(\alpha, \bar{\alpha}, x)$, $J=(\beta, \bar{\beta}, y)$, and $K=(\gamma, \bar{\gamma}, z)$ and assume locality, so that we obtain

$$
\begin{aligned}
\mathfrak{g}_{I J} & =\delta(x-y) \mathfrak{g}_{\alpha \beta}(x) \overline{\mathfrak{g}}_{\bar{\alpha} \bar{\beta}}(x) \square, \\
\mathfrak{f}_{I J K} \Phi^{J} \Phi^{K}= & \delta(x-y) \delta(x-z) \\
& \times \sum_{A, \bar{A}}\left(\mathfrak{f}_{\alpha \beta \gamma}^{A} \overline{\mathfrak{f}}_{\bar{\alpha} \bar{\alpha} \bar{\gamma} \bar{\gamma}}^{\bar{A}} \Phi^{\beta \bar{\beta}}\right)\left(\mathfrak{f}_{\alpha \beta \gamma}^{\prime A} \overline{\mathfrak{f}}_{\bar{\alpha} \bar{\beta} \bar{\gamma} \overline{\bar{\gamma}}}^{\bar{A}} \Phi^{\gamma \bar{\gamma}}\right),
\end{aligned}
$$

with $\mathfrak{g}_{\alpha \beta}$ and $\overline{\mathfrak{g}}_{\bar{\alpha} \bar{\beta}}$ graded (with respect to the ghost numbers) symmetric, and $\mathfrak{f}_{\beta \gamma}^{\delta A}$, etc., differential operators with constant coefficients. The indices $A$ and $\bar{A}$ range over the summands in $\mathfrak{f}_{I J K}$. To simplify notation, we define

$$
\mathfrak{\mathfrak { f }}_{I J K} \Phi^{J} \Phi^{K}=: \mathfrak{g}_{\alpha \delta} \overline{\mathfrak{g}}_{\bar{\alpha}} \overline{\mathfrak{\delta}}_{\beta \gamma}^{\delta} \overline{\mathfrak{f}}_{\bar{\beta} \bar{\gamma}}^{\bar{\delta}} \Phi^{\beta \bar{\beta}} \Phi^{\gamma \bar{\gamma}}
$$

Suppressing the position dependence, the Lagrangian of the theory becomes

$$
\mathcal{L}=\frac{1}{2} \Phi^{\alpha \bar{\alpha}} \mathfrak{g}_{\alpha \beta} \overline{\mathfrak{g}}_{\bar{\alpha} \bar{\beta}} \square \Phi^{\beta \bar{\beta}}+\frac{1}{3 !} \Phi^{\alpha \bar{\alpha}} \mathfrak{\mathfrak { f }}_{\alpha \beta \gamma} \overline{\mathfrak{f}}_{\bar{\alpha} \bar{\beta} \bar{\gamma}} \Phi^{\beta \bar{\beta}} \Phi^{\gamma \bar{\gamma}}
$$

where we used the shorthand $\mathfrak{f}_{\alpha \beta \gamma} \mathfrak{f}_{\bar{\alpha} \bar{\beta} \bar{\gamma}}$ for the evident expression in Eq. (3c).

Analogously, we want the BRST operator to act on left and right indices separately, and we split $Q=Q_{L}+Q_{R}$ with

$$
\begin{aligned}
Q_{L} \Phi^{\alpha \bar{\alpha}}= & \mathfrak{q}_{\mu}^{\alpha} \delta_{\bar{\mu}}^{\bar{\alpha}} \Phi^{\mu \bar{\mu}}+\frac{1}{2} \mathfrak{q}_{\mu \nu}^{\alpha} \overline{\mathfrak{f}}_{\bar{\mu} \bar{\nu}}^{\bar{\alpha}} \Phi^{\mu \bar{\mu}} \Phi^{\nu \bar{\nu}} \\
& +\frac{1}{3 !} \mathfrak{q}_{\mu \nu \kappa}^{\alpha} \overline{\mathfrak{f}}_{\bar{\mu} \bar{\nu} \bar{\kappa}}^{\alpha} \Phi^{\mu \bar{\mu}} \Phi^{\nu \bar{\nu}} \Phi^{\kappa \bar{\kappa}}
\end{aligned}
$$

where $\overline{\mathfrak{f}}_{\bar{\beta} \bar{\gamma} \bar{\delta}}^{\bar{\alpha}}=3 \overline{\mathfrak{f}}_{\bar{\varepsilon} \bar{\delta}}^{\bar{\alpha}} \overline{\mathfrak{f}}_{\bar{\beta}}^{\bar{\varepsilon}}$ and similarly for $Q_{R} \Phi$.

As an example, consider the special case of cubic Yang-Mills theory, where the $\mathfrak{g}_{\alpha \beta}$ and $\mathfrak{f}_{\beta \gamma}^{\alpha}$ are the components of the Killing form and the structure constants of a gauge algebra, respectively, while $\overline{\mathfrak{g}}_{\bar{\alpha} \bar{\beta}}$ and $\overline{\mathfrak{f}}_{\bar{\beta} \bar{\gamma}}^{\bar{\alpha}}$ are the inner product and kinematical structure constants on the full BRST field space.

To double copy means to replace the left (or right) sector with a copy of the right (or left) sector of some, not necessarily the same, theory written in the form (4), (5). If the resulting action $S$ and BRST operator $\boldsymbol{Q}$ satisfy again the relations $Q^{2}=0, Q S=0$, we obtain a consistently gauge-fixed theory ready for quantization.

It is not hard to see that $\boldsymbol{Q}_{L / R}^{2}=0$ iff $Q_{L / R}^{2}=0$; the condition $\boldsymbol{Q}_{L} \boldsymbol{Q}_{R}+\boldsymbol{Q}_{R} \boldsymbol{Q}_{L}=0$ may induce further conditions. For Yang-Mills theory, one readily computes that CK duality suffices for the condition $Q S=0$. If CK duality fails to hold up to certain terms, then $Q S=0$ also fails to hold up to the same terms, possibly multiplied by other fields and their derivatives. (Mathematically, the terms describing the failure of CK duality generate an ideal in the algebra of fields and their derivatives. The expressions $Q S$ and $Q^{2}$ take values in this ideal.)

Preliminary observations. - We are interested in perturbative aspects and omit any nonperturbative issues. Also, we are interested in $n$-point amplitudes up to $\ell$ loops for $n$ and $\ell$ finite. Thus, there is always a number $N \in \mathbb{N}$ so that monomials of degree $m>N$ can be neglected in the Lagrangian. We always use the term "amplitude" for onshell states and the term "correlator" for off-shell states.

Although the quantization of Yang-Mills theory does not require it, it is convenient to start from the BV form [57] of the Yang-Mills Lagrangian on Minkowski space, using canonical notation for all fields,

$\mathcal{L}_{\mathrm{YM}}:=-\frac{1}{4} F_{\mu \nu}^{a} F^{a \mu \nu}+A_{\mu}^{+a}\left(\nabla^{\mu} c\right)^{a}+\frac{g}{2} f_{b c}^{a} c^{+a} c^{b} c^{c}+b^{a} \bar{c}^{+a}$,

with $g$ the Yang-Mills coupling constant. We use the gauge fixing fermion $\Psi:=\Psi_{0}+\Psi_{1}$ with

$\Psi_{0}:=\int d^{d} x \bar{c}^{a}\left(\frac{\xi}{2} b^{a}-\partial^{\mu} A_{\mu}^{a}\right), \quad \Psi_{1}:=\int d^{d} x \bar{c}^{a} \psi^{a}$,

where $\psi^{a}$ is of ghost number 0 and depends at least quadratically on the fields and their derivatives. We obtain the gauge-fixed Lagrangian

$$
\begin{aligned}
\mathcal{L}_{\mathrm{YM}}^{\mathrm{gf}}= & -\frac{1}{4} F_{\mu \nu}^{a} F^{a \mu \nu}-\bar{c}^{a} \partial^{\mu}\left(\nabla_{\mu} c\right)^{a}+\frac{\xi}{2}\left(b^{a}\right)^{2}-b^{a} \partial^{\mu} A_{\mu}^{a} \\
& +\frac{\delta \Psi_{1}}{\delta A_{\mu}^{a}}\left(\nabla_{\mu} c\right)^{a}+\frac{g}{2} f_{b c}^{a} \frac{\delta \Psi_{1}}{\delta c^{a}} c^{b} c^{c}+b^{a} \frac{\delta \Psi_{1}}{\delta \bar{c}^{a}} .
\end{aligned}
$$

For $\psi^{a}=0$, we recover the $R_{\xi}$ gauges. The BRST transformations are 


$$
\begin{aligned}
& Q_{\mathrm{YM}} c^{a}:=-\frac{g}{2} f_{b c}^{a} c^{b} c^{c}, \quad Q_{\mathrm{YM}} A_{\mu}^{a}:=\left(\nabla_{\mu} c\right)^{a}, \\
& Q_{\mathrm{YM}} b^{a}:=0, \quad Q \bar{c}^{a}:=b^{a},
\end{aligned}
$$

satisfying $Q_{\mathrm{YM}}^{2}=0$ off shell.

The nonphysical fields enlarge the one-particle field space of asymptotic on-shell states by four types of states: the two unphysical polarizations of the gluon, called forward and backward and denoted by $A^{\uparrow}$ and $A^{\downarrow}$, and the ghost and antighost states [45]. All amplitudes will be built from the $n$-particle form of this BRST-extended onshell field space, which carries an action of the linearization of Eq. (9) denoted by $Q_{\mathrm{YM}}^{\text {lin }}$. The physical polarizations are singlets, $Q_{\mathrm{YM}}^{\operatorname{lin}} A_{\text {phys }}=0$, and we have two more doublets:

$$
A \stackrel{Q_{\mathrm{YM}}^{\mathrm{lin}}}{\longrightarrow} c \quad \text { and } \quad \bar{c} \stackrel{Q_{\mathrm{YM}}^{\mathrm{lin}}}{\longrightarrow} b=\frac{1}{\xi} \partial^{\mu} A_{\mu}^{\downarrow}+\cdots,
$$

where the ellipsis indicates terms that arise from $\Psi_{1}$.

Observation 1: The set of connected correlation functions is BRST invariant because they can be written as linear combinations of products of correlation functions.

Crucial to our discussion are the supersymmetric Ward identities generated by the BRST operator. We start with the on-shell form, see, e.g., Refs. [58,59]. Since the free vacuum is invariant under the action of $Q_{\mathrm{YM}}^{\text {lin }}$, we have the following on-shell Ward identities:

$$
0=\left\langle 0\left|\left[Q_{\mathrm{YM}}, \mathcal{O}_{1} \cdots \mathcal{O}_{n}\right]\right| 0\right\rangle .
$$

We now consider the on-shell Ward identity for $\mathcal{O}_{1} \cdots \mathcal{O}_{n}=A^{\uparrow} \bar{c}(c \bar{c})^{k} A_{\text {phys }}^{n-2 k-2}$ and obtain

$$
\left\langle 0\left|(c \bar{c})^{k+1} A_{\text {phys }}^{n-2 k-2}\right| 0\right\rangle \sim\left\langle 0\left|A^{\uparrow}(c \bar{c})^{k} b A_{\text {phys }}^{n-2 k-2}\right| 0\right\rangle .
$$

Thus,

Observation 2: Any amplitude with $k+1$ ghost-antighost pairs and all gluons transversely polarized is given by a sum of amplitudes with $k$ ghost pairs.

From the construction of amplitudes via Feynman diagrams, it follows that we also have the following onshell Ward identity for an approximate BRST symmetry.

Observation 3: Suppose that $Q S=0$ and $Q^{2}=0$ only on shell. Then, we still have Eq. (11) together with a corresponding identification of amplitudes with $k+1$ ghost-antighost pairs and all gluons transversely polarized and a sum of amplitudes with $k$ ghost pairs.

We shall also need the off-shell form of these Ward identities,

$$
\begin{aligned}
\partial^{\mu}\left\langle j_{\mu}(x) \mathcal{O}_{1}\left(x_{1}\right) \cdots \mathcal{O}_{n}\left(x_{n}\right)\right\rangle \\
\quad=\sum_{i=1}^{n} \mp \delta\left(x-x_{i}\right)\left\langle\left(Q \mathcal{O}_{i}\left(x_{i}\right)\right) \Pi_{j \neq i} \mathcal{O}_{j}\left(x_{j}\right)\right\rangle,
\end{aligned}
$$

where $j_{\mu}$ is the BRST current. The left-hand side vanishes after integration over $x$, and using observation 1 , we can restrict to connected correlators at a particular order in the coupling constant $g$ and then further to lowest order in $\hbar$, i.e., to tree level. Consider now operators $\mathcal{O}_{i}\left(x_{i}\right)$ for those restricted Ward identities which are linear in the fields.

Observation 4: The on-shell relations between tree amplitudes from observation 2 induced by Eq. (11) extend to (off-shell) tree-level connected correlators. For example,

$$
\begin{aligned}
& \left\langle A_{\mu}\left(x_{1}\right) b\left(x_{2}\right) A_{\nu}\left(x_{3}\right)\right\rangle \\
& =\left\langle\partial_{\mu} c\left(x_{1}\right) \bar{c}\left(x_{2}\right) A_{\nu}\left(x_{3}\right)\right\rangle+\left\langle A_{\mu}\left(x_{1}\right) \bar{c}\left(x_{2}\right) \partial_{\nu} c\left(x_{3}\right)\right\rangle .
\end{aligned}
$$

Next, we make the following three general observations:

Observation 5: If two field theories have the same tree amplitudes, then the minimal models of their $L_{\infty}$-algebras coincide, cf. Refs. [55,56]. If they have the same field content and kinetic parts, then they are related by a local (invertible) field redefinition.

Observation 6: Two field theories are quantum equivalent, if all their correlators agree. Since correlators can be glued together from tree-level correlators (up to regularization issues), it suffices if the latter agree.

Observation 7: A shift of a field by products of fields and their derivatives which do not involve the field itself does not change the path integral measure. Local field redefinitions that are trivial at linear order produce a Jacobian that is regulated to unity in dimensional regularization [60-62], see also Ref. [63]. Therefore, they preserve quantum equivalence.

In our constructions, we will also exploit the possibility of adjusting our choice of gauge. Performing shifts $b^{a} \mapsto$ $b^{a}+X^{a}$ and $\Psi_{1} \mapsto \Psi_{1}+\Xi_{1}$ with $\Xi_{1}:=\int d^{d} x \bar{c}^{a} Y^{a}$ induces a shift of (8) by

$$
\begin{aligned}
& \frac{\xi}{2}\left(X^{a}\right)^{2}+X^{a}\left(\xi b^{a}-\partial^{\mu} A_{\mu}^{a}\right)+X^{a} \frac{\delta \Psi_{1}}{\delta \bar{c}^{a}} \\
& \quad+\frac{\delta \Xi_{1}}{\delta A_{\mu}^{a}}\left(\nabla_{\mu} c\right)^{a}+\frac{g}{2} f_{b c}^{a} \frac{\delta \Xi_{1}}{\delta c^{a}} c^{b} c^{c}+\left(b^{a}+X^{a}\right) \frac{\delta \Xi_{1}}{\delta \bar{c}^{a}} .
\end{aligned}
$$

If $X^{a}$ is independent of the NL field $b^{a}$, this modification preserves the theory at the quantum level by observation 7 . Furthermore, if $X^{a}$ is at least quadratic in the fields, this transformation preserves the action of $Q_{\mathrm{YM}}^{\text {lin }}$ on the BRSTextended on-shell field space.

Consider now the special case $\psi^{a}=0$ and $X^{a}$ independent of $b^{a}$ and fix $Y^{a}$ iteratively such that the terms linear in $b^{a}$ of Eq. (15) vanish:

$$
\xi X^{a}+\frac{\delta \Xi_{1}}{\delta \bar{c}^{a}}=\xi X^{a}+Y^{a}+\bar{c}^{b} \frac{\partial Y^{a}}{\partial \bar{c}^{b}}+\cdots=0 .
$$

This leads to the following observation: 
Observation 8: Terms in the Lagrangian of the form $\left(\partial^{\mu} A_{\mu}\right)^{a} X^{a}$ with $X^{a}$ at least quadratic in the fields and their derivatives but independent of the NL field can be removed in $R_{\xi}$ gauges by shifting the NL field. This creates additional terms (15) which are at least of fourth order and preserve the amplitudes by observation 7 .

Observation 9: Terms in the action that are proportional to a NL field can be absorbed by choosing a suitable term $\psi^{a}$. This leaves the physical sector invariant but it may modify the ghost sector. Because NL fields appear as trivial pairs in the BV action, it is not hard to see that this extends to general gauge theories, e.g., with several NL fields and ghosts for ghosts.

We also make the following three observations regarding the double copy.

Observation 10: The tree amplitudes of Yang-Mills theory can be written in CK-dual form [4-12].

Observation 11: For amplitudes in CK-dual form, there is a corresponding local, cubic, and physically equivalent Lagrangian whose partial amplitudes produce the kinematical numerators [39].

Observation 12: Double copying the Yang-Mills tree amplitudes in CK-dual form yields the tree amplitudes of $\mathcal{N}=0$ supergravity [1-3].

CK-dual Yang-Mills theory.-In order to BRSTLagrangian double copy Yang-Mills theory, we first must bring its action into the normalized form (4). Our goal will be to construct abstractly a Lagrangian that guarantees tree-level CK duality for the BRST-extended on-shell field space.

CK duality of the Feynman diagrams for the field space of physical gluons can be guaranteed by adding terms to the Lagrangian $[3,39]$ and subsequently strictifying these, i.e., introducing a set of auxiliary fields such that all interaction vertices are cubic. This strictification is mostly determined by the color and momentum structure of the additional terms in the Lagrangian.

It remains to ensure CK duality for tree amplitudes involving ghosts or backward polarized gluon states, which we do by introducing compensating terms, preserving quantum equivalence. (Forward polarized gluons can be absorbed by residual gauge transformations and therefore do not appear in the Lagrangian. Thus, they cannot contribute to $\mathrm{CK}$ duality violations.)

We implement the necessary changes iteratively for $n$-point amplitudes, starting with $n=4$. We can compensate for CK duality violations due to backward polarized gluons, which can be done by introducing terms of the form $\left(\partial^{\mu} A_{\mu}\right)^{a} X^{a}$. By observation 8 , we can produce such terms, preserving quantum equivalence, and we immediately compensate for the additional terms linear in the NL field using observation 9. Since we perform all shifts at the level of the BV action and the gauge fixing fermion, the resulting action is automatically BRST invariant and its amplitudes are CK dual for external legs of ghost number 0.
By observation 2, these amplitudes fully determine all amplitudes with ghosts and antighosts on external legs. Moreover, the CK-dual form of the former can be copied over to the latter, by literally copying trivalent Feynman diagrams for the gluon modes linked by the BRST symmetry to the ghost-antighost pairs. We do this iteratively in the number of ghost-antighost pairs. The consistency of the copying process is guaranteed by the full BRST symmetry of the action. We then use observation 12 to turn these CK-dual amplitudes for arbitrary ghost number into a local, cubic, and BRST-invariant Lagrangian.

The resulting Lagrangian $\mathcal{L}_{\mathrm{YM}}^{\mathrm{CK}}$ is of the form (4) and quantum equivalent to the Lagrangian $\mathcal{L}_{\mathrm{YM}}$ given in Eq. (8).

The BRST-Lagrangian double copy of Yang-Mills Theory. -We now turn to the $\mathcal{N}=0$ supergravity side. The gauge-fixed BRST Lagrangian $\mathcal{L}_{\mathcal{N}=0}$ of this theory is readily constructed. The following two diagrams concisely summarize the theory's field content from the perspective of the double copy, describing the symmetrized and antisymmetrized tensor products of two copies of the BRST Yang-Mills fields:
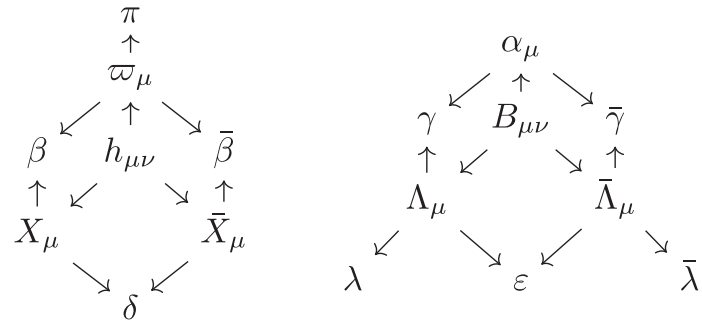

Here, the physical fields of ghost number 0 are $h_{\mu \nu}$ (containing the metric perturbation about the Minkowski vacuum and the dilaton) and $B_{\mu \nu}$ (the Kalb-Ramond twoform). Ghost number increases by column from left to right, and all vector or form indices are made explicit. The arrows indicate factorization relations between the various fields [52]. In addition to the expected BRST field content, we have two trivial BV pairs $(\delta, \beta)$ and $(\bar{\beta}, \pi)$, see, e.g., Ref. [64] for the same fields in a different context. For more details, see Ref. [52] as well as Refs. [44,46-49].

The double copy of $Q_{\mathrm{YM}}$ and $\mathcal{L}_{\mathrm{YM}}^{\mathrm{CK}}$ yields a BRST operator $\boldsymbol{Q}$ which satisfies $\boldsymbol{Q}^{2}=0$ on shell and a Lagrangian $\mathcal{L}$ for the field content (17). The latter is quantum equivalent to the manifestly $\mathrm{CK}$ dual, cubic or strict form $\mathcal{L}_{\mathcal{N}=0}^{\text {st }}$ of $\mathcal{N}=0$ supergravity obtained from observation 11: (i) Kinematic equivalence.-The two kinematic Lagrangians are equivalent and linked by evident suitable field redefinitions [52]. The existence of such a field redefinition is ensured by the linear double copy BRST operator $\boldsymbol{Q}^{\text {lin }}[44,48]$, which is equivalent to the linear BRST operator $Q_{\mathcal{N}=0}^{\text {stlin }}$ and annihilates the quadratic double copy Lagrangian [52]. We implement the field redefinition on $\mathcal{L}_{\mathcal{N}=0}^{\text {st }}$, obtaining $\mathcal{L}_{\mathcal{N}=0}^{\text {st, } 1}$. (ii) Ghost 
number 0, partly.- Since the classical Yang-Mills action was written in a form with purely cubic, local interactions with manifest CK duality to all points, the tree amplitudes of $\mathcal{L}$ for physical fields match those of $\mathcal{L}_{\mathcal{N}=0}^{\text {st, }}$, cf. observation 12. The amplitudes for auxiliaries of ghost number 0 are determined by collinear limits of amplitudes of physical fields and thus also agree between the theories. By observation 5, we can implement a field redefinition $\mathcal{L}_{\mathcal{N}=0}^{\text {st, } 1} \rightarrow \mathcal{L}_{\mathcal{N}=0}^{\text {st,2 }}$ such that the interaction vertices of $\mathcal{L}_{\mathcal{N}=0}^{\text {st, } 2}$ and $\mathcal{L}$ agree for physical and auxiliary fields of ghost number 0 to any finite order. For these fields, also the tree-level correlators agree, because the field redefinitions preserve quantum equivalence by observation 7. (iii) Gauge fixing sector.-The difference between $\mathcal{L}$, after integrating out all auxiliary fields, and $\mathcal{L}_{\mathcal{N}=0}^{\text {st, } 2}$ proportional to any of the NL-like fields $\left(\beta, \bar{\beta}, \varpi_{\mu}, \pi, \gamma, \alpha_{\mu}, \bar{\gamma}\right)$ can be absorbed in a choice of gauge fixing which will only create new terms in the ghost sector, cf. observation 9 for all fields except for $\beta$, which requires a slightly different treatment [52]. We implement this new gauge fixing, and take over the strictification from $\mathcal{L}$, obtaining $\mathcal{L}_{\mathcal{N}=0}^{\text {st,3 }}$ together with a BRST operator $Q_{\mathcal{N}=0}^{\mathrm{st}, 3}$. (iv) Ghost sector.-Starting from the latter, we now use observation 2 to copy over the CK-dual form of the amplitudes with external legs labeled exclusively by fields of ghost number 0 to a CK-dual form of amplitudes with ghost-antighost pairs on external legs. This proceeds just as in the case of Yang-Mills theory and consistency is again guaranteed by full BRST symmetry of the action. By observation 12, we can then turn these CKdual amplitudes into a local, cubic, and CK duality manifesting Lagrangian $\mathcal{L}_{\mathcal{N}=0}^{\mathrm{CK}}$ physically equivalent to $\mathcal{L}_{\mathcal{N}=0}^{\text {st, } 3}$ and thus to $\mathcal{L}_{\mathcal{N}=0}$.

Both $\mathcal{L}$ and $\mathcal{L}_{\mathcal{N}=0}^{\mathrm{CK}}$ are local and have the same field content. The tree-level correlators involving physical and NL fields agree. Using the approximate Ward identities, cf. Observation 3, and the fact that $Q^{\text {lin }}$ and $Q_{\mathcal{N}=0}^{\mathrm{CK} \text {,lin }}$ agree, we deduce that all tree amplitudes involving ghosts and antighost pairs agree, too. By construction, this agreement extends to individual onshell Feynman diagrams, between the strictifications $\mathcal{L}$ and $\mathcal{L}_{\mathcal{N}=0}^{\mathrm{CK}}$, even for auxiliary fields: we can iteratively split off external vertices with two external legs, exposing Feynman diagrams with onshell external but off-shell auxiliary fields. Up to a field redefinition of the auxiliaries, these also must agree.

The only potential remaining difference between $\mathcal{L}$ and $\mathcal{L}_{\mathcal{N}=0}^{\mathrm{CK}}$ is then interaction terms containing $\square \Gamma$ and $\square \bar{\Gamma}$ terms for $\Gamma$ a ghost field. Going through the construction, one can argue that such terms, if they are there, have to appear in the same way in $\mathcal{L}$ and $\mathcal{L}_{\mathcal{N}=0}^{\mathrm{CK}}$. Alternatively, one can show that both theories satisfy the same Ward identities for tree-level correlators, rendering them quantum equivalent by observation 6 . The simplest argument, however, is to use observation 5 to note that both theories are related by a local field redefinition. Observation 7 then implies that both theories are quantum equivalent.

No additional research data beyond the data presented and cited in this work are needed to validate the research findings in this work.

We gratefully acknowledge stimulating conversations with Johannes Brödel, Michael Duff, Henrik Johansson, Silvia Nagy, Jim Stasheff, and Alessandro Torrielli. L. B., H. K., and C.S. were supported by the Leverhulme Research Project Grant No. RPG-2018-329 "The Mathematics of M5-Branes." B. J. was supported by the GAČR Grant No. EXPRO 19-28268X and thanks MPIM Bonn for hospitality. T. M. was partially supported by the EPSRC Grant No. EP/N509772.

\footnotetext{
*1.borsten@hw.ac.uk

†ranislav.jurco@gmail.com

"hk55@hw.ac.uk

${ }^{\S}$ t.macrelli@surrey.ac.uk

c.saemann@hw.ac.uk

"m.wolf@surrey.ac.uk
}

[1] Z. Bern, J. J. M. Carrasco, and H. Johansson, New relations for gauge-theory amplitudes, Phys. Rev. D 78, 085011 (2008).

[2] Z. Bern, J. J. M. Carrasco, and H. Johansson, Perturbative Quantum Gravity as a Double Copy of Gauge Theory, Phys. Rev. Lett. 105, 061602 (2010).

[3] Z. Bern, T. Dennen, Y.-t. Huang, and M. Kiermaier, Gravity as the square of gauge theory, Phys. Rev. D 82, 065003 (2010).

[4] S. Stieberger, Open \& closed vs. pure open string disk amplitudes, arXiv:0907.2211.

[5] N. E. J. Bjerrum-Bohr, P. H. Damgaard, and P. Vanhove, Minimal Basis for Gauge Theory Amplitudes, Phys. Rev. Lett. 103, 161602 (2009).

[6] Y. Jia, R. Huang, and C.-Y. Liu, U(1)-decoupling, Kleiss-Kuijf and Bern-Carrasco-Johansson relations in $\mathcal{N}=4$ super Yang-Mills, Phys. Rev. D 82, 065001 (2010).

[7] N. E. J. Bjerrum-Bohr, P. H. Damgaard, T. Søndergaard, and P. Vanhove, The momentum kernel of gauge and gravity theories, J. High Energy Phys. 01 (2011) 001.

[8] B. Feng, R. Huang, and Y. Jia, Gauge amplitude identities by on-shell recursion relation in S-matrix program, Phys. Lett. B 695, 350 (2011).

[9] Y.-X. Chen, Y.-J. Du, and B. Feng, A proof of the explicit minimal-basis expansion of tree amplitudes in gauge field theory, J. High Energy Phys. 02 (2011) 112.

[10] C. R. Mafra, O. Schlotterer, and S. Stieberger, Explicit BCJ numerators from pure spinors, J. High Energy Phys. 07 (2011) 092.

[11] Y.-J. Du and C.-H. Fu, Explicit BCJ numerators of nonlinear sigma model, J. High Energy Phys. 09 (2016) 174.

[12] S. Mizera, Kinematic Jacobi Identity is a Residue Theorem: Geometry of Color-Kinematics Duality for Gauge and Gravity Amplitudes, Phys. Rev. Lett. 124, 141601 (2020). 
[13] J. J. M. Carrasco, Gauge and gravity amplitude relations, in Proceedings, Theoretical Advanced Study Institute in Elementary Particle Physics: Journeys Through the Precision Frontier: Amplitudes for Colliders (TASI 2014), Boulder, Colorado, 2014 [arXiv:1506.00974].

[14] Z. Bern, J. J. Carrasco, M. Chiodaroli, H. Johansson, and R. Roiban, The duality between color and kinematics and its applications, arXiv:1909.01358.

[15] L. Borsten, Gravity as the square of gauge theory: A review, Riv. Nuovo Cimento 43, 97 (2020).

[16] Z. Bern, J. J. M. Carrasco, L. J. Dixon, H. Johansson, and R. Roiban, The complete four-loop four-point amplitude in $\mathcal{N}=4$ super-Yang-Mills theory, Phys. Rev. D 82, 125040 (2010).

[17] J. J. M. Carrasco and H. Johansson, Five-point amplitudes in $\mathcal{N}=4$ Super-Yang-Mills theory and $\mathcal{N}=8$ supergravity, Phys. Rev. D 85, 025006 (2012).

[18] Z. Bern, C. Boucher-Veronneau, and H. Johansson, $\mathcal{N} \leq 4$ supergravity amplitudes from gauge theory at one loop, Phys. Rev. D 84, 105035 (2011).

[19] C. Boucher-Veronneau and L. J. Dixon, $\mathcal{N} \leq 4$ supergravity amplitudes from gauge theory at two loops, J. High Energy Phys. 12 (2011) 046.

[20] Z. Bern, S. Davies, T. Dennen, and Y.-t. Huang, Absence of Three-Loop Four-Point Ultraviolet Divergences in $\mathcal{N}=4$ Supergravity, Phys. Rev. Lett. 108, 201301 (2012).

[21] Z. Bern, S. Davies, T. Dennen, and Y.-t. Huang, Ultraviolet cancellations in half-maximal supergravity as a consequence of the double-copy structure, Phys. Rev. D 86, 105014 (2012).

[22] S. Oxburgh and C. D. White, BCJ duality and the double copy in the soft limit, J. High Energy Phys. 02 (2013) 127.

[23] Z. Bern, J. J. M. Carrasco, L. J. Dixon, H. Johansson, and R. Roiban, Simplifying multiloop integrands and ultraviolet divergences of gauge theory and gravity amplitudes, Phys. Rev. D 85, 1050142012.

[24] Y.-J. Du and H. Luo, On general BCJ relation at one-loop level in Yang-Mills theory, J. High Energy Phys. 01 (2013) 129.

[25] E. Y. Yuan, Virtual color-kinematics duality: 6-pt 1-loop MHV amplitudes, J. High Energy Phys. 05 (2013) 070.

[26] Z. Bern, S. Davies, T. Dennen, A. V. Smirnov, and V. A. Smirnov, Ultraviolet Properties of $\mathcal{N}=4$ Supergravity at Four Loops, Phys. Rev. Lett. 111, 231302 (2013).

[27] R. H. Boels, R. S. Isermann, R. Monteiro, and D. O'Connell, Colour-kinematics duality for one-loop rational amplitudes, J. High Energy Phys. 04 (2013) 107.

[28] Z. Bern, S. Davies, T. Dennen, Y.-t. Huang, and J. Nohle, Color-kinematics duality for pure Yang-Mills and gravity at one and two loops, Phys. Rev. D 92, 045041 (2015).

[29] Z. Bern, S. Davies, and T. Dennen, The ultraviolet structure of half-maximal supergravity with matter multiplets at two and three loops, Phys. Rev. D 88, 065007 (2013).

[30] Z. Bern, S. Davies, and T. Dennen, The ultraviolet critical dimension of half-maximal supergravity at three loops, arXiv:1412.2441.

[31] Z. Bern, S. Davies, and T. Dennen, Enhanced ultraviolet cancellations in $\mathcal{N}=5$ supergravity at four loop, Phys. Rev. D 90, 105011 (2014).

[32] C. R. Mafra and O. Schlotterer, Towards one-loop SYM amplitudes from the pure spinor BRST cohomology, Fortschr. Phys. 63, 105 (2015).
[33] C. R. Mafra and O. Schlotterer, Two-loop five-point amplitudes of super Yang-Mills and supergravity in pure spinor superspace, J. High Energy Phys. 10 (2015) 124.

[34] H. Johansson, G. Klin, and G. Mogull, Two-loop supersymmetric QCD and half-maximal supergravity amplitudes, J. High Energy Phys. 09 (2017) 019.

[35] Z. Bern, J. J. M. Carrasco, W.-M. Chen, H. Johansson, R. Roiban, and M. Zeng, The five-loop four-point integrand of $\mathcal{N}=8$ supergravity as a generalized double copy, Phys. Rev. D 96, 126012 (2017).

[36] Z. Bern, J. J. Carrasco, W.-M. Chen, A. Edison, H. Johansson, J. Parra-Martinez, R. Roiban, and M. Zeng, Ultraviolet properties of $\mathcal{N}=8$ supergravity at five loops, Phys. Rev. D 98, 086021 (2018).

[37] R. Monteiro and D. O'Connell, The kinematic algebra from the self-dual sector, J. High Energy Phys. 07 (2011) 007.

[38] N. E. J. Bjerrum-Bohr, P. H. Damgaard, R. Monteiro, and D. O'Connell, Algebras for amplitudes, J. High Energy Phys. 06 (2012) 061.

[39] M. Tolotti and S. Weinzierl, Construction of an effective Yang-Mills Lagrangian with manifest BCJ duality, J. High Energy Phys. 07 (2013) 111.

[40] R. Monteiro and D. O'Connell, The kinematic algebras from the scattering equations, J. High Energy Phys. 03 (2014) 110.

[41] C.-H. Fu and K. Krasnov, Colour-kinematics duality and the Drinfeld double of the Lie algebra of diffeomorphisms, J. High Energy Phys. 01 (2017) 075.

[42] C. Cheung and C.-H. Shen, Symmetry for Flavor-Kinematics Duality from Action, Phys. Rev. Lett. 118, 121601 (2017).

[43] G. Chen, H. Johansson, F. Teng, and T. Wang, On the kinematic algebra for BCJ numerators beyond the MHV sector, J. High Energy Phys. 11 (2019) 055.

[44] L. Borsten and S. Nagy, The pure BRST Einstein-Hilbert Lagrangian from the double-copy to cubic order, J. High Energy Phys. 07 (2020) 093.

[45] T. Kugo and I. Ojima, Manifestly covariant canonical formulation of Yang-Mills field theories. I. General formalism, Prog. Theor. Phys. 60, 1869 (1978).

[46] A. Anastasiou, L. Borsten, M. J. Duff, L. J. Hughes, and S. Nagy, Yang-Mills Origin of Gravitational Symmetries, Phys. Rev. Lett. 113, 231606 (2014).

[47] L. Borsten, On $D=6, \mathcal{N}=(2,0)$ and $\mathcal{N}=(4,0)$ theories, Phys. Rev. D 97, 066014 (2018).

[48] A. Anastasiou, L. Borsten, M. J. Duff, S. Nagy, and M. Zoccali, Gravity as Gauge Theory Squared: A Ghost Story, Phys. Rev. Lett. 121, 211601 (2018).

[49] M. Zoccali, Supergravity as Yang-Mills squared, Ph.D. thesis, Imperial College London, 2018.

[50] L. Borsten, I. Jubb, V. Makwana, and S. Nagy, Gauge $\times$ gauge on spheres, J. High Energy Phys. 06 (2020) 096

[51] A. Luna, S. Nagy, and C. White, The convolutional double copy: A case study with a point, J. High Energy Phys. 09 (2020) 062.

[52] L. Borsten, B. Jurčo, H. Kim, T. Macrelli, C. Saemann, and M. Wolf, Double copy from homotopy algebras, arXiv: 2102.11390 .

[53] Z. Bern, J. J. Carrasco, W.-M. Chen, H. Johansson, and R. Roiban, Gravity Amplitudes as Generalized Double Copies, Phys. Rev. Lett. 118, 181602 (2017). 
[54] E. Casali, S. Mizera, and P. Tourkine, Loop amplitudes monodromy relations and color-kinematics duality, J. High Energy Phys. 03 (2021) 048.

[55] B. Jurčo, L. Raspollini, C. Saemann, and M. Wolf, $L_{\infty}$-algebras of classical field theories and the BatalinVilkovisky formalism, Fortschr. Phys. 67, 1900025 (2019).

[56] T. Macrelli, C. Saemann, and M. Wolf, Scattering amplitude recursion relations in BV quantizable theories, Phys. Rev. D 100, 045017 (2019).

[57] I. A. Batalin and G. A. Vilkovisky, Gauge algebra and quantization, Phys. Lett. 102B, 27 (1981).

[58] H. Elvang and Y.-t. Huang, Scattering amplitudes, arXiv: 1308.1697.
[59] H. Elvang and Y.-t. Huang, Scattering Amplitudes in Gauge Theory and Gravity (Cambridge University Press, Cambridge, England, 2015).

[60] I. Tyutin, Once again on the equivalence theorem, Phys. At. Nucl. 65, 194 (2002).

[61] G. 't Hooft and M. Veltman, Diagrammar, NATO Sci. Ser. B 4, 177 (1974).

[62] G. Leibbrandt, Introduction to the technique of dimensional regularization, Rev. Mod. Phys. 47, 849 (1975).

[63] M. Henneaux and C. Teitelboim, Quantization of Gauge Systems (Princeton University Press, Princeton, NJ, 1992).

[64] L. Baulieu, Unimodular gauge in perturbative gravity and supergravity, Phys. Lett. B 808, 135591 (2020). 